\title{
Distributed Data Processing in Vehicular Networks using Mobile
}

\section{Agents}

\author{
Óscar Urra, Sergio Ilarri \\ Sistemas de Información Distribuidos (SID) \\ Instituto de Investigación en Ingeniería de Aragón (I3A). \\ Universidad de Zaragoza, Mariano Esquillor s/n, 50018, Zaragoza, Spain. \\ Tel. +34-976762707, Fax +34-976762043, e-mail: ourra@ita.es
}

\begin{abstract}
In a vehicular network, vehicles can exchange interesting information (e.g., about accidents, traffic status, etc.) using short-range wireless communications. Besides, the vehicles can be equipped with additional sensors that can directly obtain data from the environment. How to efficiently process and collect these data is an open problem. We argue that mobile agent technology could be helpful.
\end{abstract}

\section{Introduction}

A vehicular ad-hoc network (or VANET) [4] consists of a number of vehicles traveling across the roads of a certain area. Those vehicles can share data relevant for the drivers by using short-range communication (such as Wi-Fi o UWB).

Given the mobile nature of the vehicles and their presence along wide geographic areas, these vehicles could be used as a sensing platform to obtain interesting data if appropriate sensors (e.g., to measure the $\mathrm{CO} 2$ or other pollution gases) are installed on them. However, an efficient and effective collection and processing of those data can be a very difficult task, since the communications are not stable due to their short-range and to the constant movement of the vehicles.

To overcome these difficulties, we propose the use of mobile agents [1], which are software entities that can move from one execution environment to another using a communication network. Mobile agents are autonomous, they can tolerate network disconnections and transmission failures, and they can be programmed to process specific data and get results from them according to our interests.

\section{Application Scenario}

The final goal is the retrieval of information from the VANET, by querying the raw data that is constantly retrieved by the vehicles and stored locally on them. The amount of data that can be gathered over time can be quite significant, and it would be very difficult to transfer them to a centralized place for later processing, due to a number of problems. First, we do not contemplate the use of mobile phone based networks (such as 3G or 4G), since they have an economic cost and they have also limitations in their coverage, transmission speed, and even sometimes the amount of data that can be transferred monthly. The second problem is that the collected data can be large and it would be very inefficient to transfer all of them when it is likely that only a small fraction is relevant. Finally, another concern is related to privacy, since the data collected can be of sensitive nature (e.g., the position of the car at certain hours).

To accomplish the query processing task we consider three phases. First, the user creates a query specifying the information that he/she wants to obtain; for example, the amount of $\mathrm{CO} 2$ in the city center at rush hours, the presence of free parking lots in the streets near a commercial area, etc. Then, the query is sent to the VANET and the data stored on the vehicles present in the area of interest are filtered locally to get a partial answer. Finally, all the partial solutions are returned to the query originator and the answer is obtained by merging.

However, a VANET presents challenges due to the short transmission range (around $200 \mathrm{~m}$, due to interferences or obstacles that block the signal [3]). This may require appropriate multi-hop routing.

\section{Use of Mobile Agents}

To deal with the problems of VANETs, we propose the use of mobile agents. Mobile agents are 
programs that run in an execution environment and that, at any moment, can pause their execution, move through a network to another device, and resume their execution in the destination. That execution environment is lightweight software called the mobile agent platform, and must be present in all the participating devices in the network. The platform provides the mobile agents their ability to move from one place to another, besides other services such as locating other platforms present within the coverage area at any moment or communicating with other agents in the same platform [2].

Besides their mobility and autonomy, mobile agents can be programmed to perform any required task. In our VANET scenario, they can use different algorithms to reach their destination (the interest area) by hopping from one vehicle to another [5]. Although it is not strictly necessary, these algorithms (called hop strategies) can use publicly available digital maps (such as OpenStreetMaps, http://www.openstreemaps.org) to increase their efficiency and effectiveness. The main challenge is that the routes followed by the vehicles can be unpredictable, so once the mobile agent arrives at a certain vehicle, it must keep evaluating constantly its position to hop to other nearby cars if they seem more promising than the current one to reach the destination sooner. Using data from other vehicles (such as their position, heading, and distance to the destination), the mobile agent can decide if it is worth it to hop to the other car or remain in the same one.

Applying the approach based on mobile agents to the 3-stage process described before, it would take the following form. In the first phase, the query is created and a mobile agent travels to the interest area according to a hop strategy using intermediate vehicles as relays until it reaches the destination. Once there, the data collection phase begins: the agent hops from car to car within the area to collect interesting data. If the mobile agent leaves the area due to the unpredictability of the vehicle's movements, it will try to return as soon as possible to continue the process (again, by hopping). Finally, the agent comes back to the query originator with the answer, again by applying a hopping strategy. So agents move both wirelessly (network communication) and physically (by keeping themselves executing on the cars and benefiting from the vehicles' locomotion).

\section{Conclusions and Future Work}

The retrieval of relevant information from a VANET can be challenging. The use of mobile agents provides a number of advantages over other traditional centralized approaches; so, we presented a proposal in [5]. However there also exist some issues that need to be addressed. First, due to the complexity of the environment, the whole process may take an amount of time that is only affordable for delay-tolerant queries. Another problem arises when the density of vehicles in the area is low, which can lead to situations where a mobile agent cannot found appropriate vehicles to hop to and can "get lost" if it moves too far away from the intended target area. As future work, we are working on finding solutions to these issues as well as on enhancing the efficiency of the query processing.

\section{REFERENCES}

[1]. MILOJICIC, D., DOUGLIS, F. and WHEELER, R. Mobility: processes, computers, and agents. New York: ACM, 1999.

[2]. TRILLO, R., ILARRI, S., and MENA, E. Comparison and performance evaluation of mobile agent platforms. In: Third International Conference on Autonomic and Autonomous Systems (ICAS'07). [Athens]: IEEE, 2007, p. 41.

[3]. MARTINEZ, F.J., FOGUE, M., TOH, C.K., CANO, J.C., CALAFATE, C.T. and MANZONI, P. Computer simulations of VANETs using realistic city topologies. Wireless Personal Communications. 2013, 69(2), 639-663.

[4]. OLARIU, S. and WEIGLE, M.C. Vehicular Networks: From Theory to Practice. London: Chapman \& Hall/CRC, 2009.

[5]. URRA, O. and ILARRI, S. Using mobile agents in vehicular networks for data processing. In: 14th International Conference on Mobile Data Management (MDM 2013). [Milan]: IEEE, 2013. Vol. 2, pp. 11-14. 